\title{
Finite-Time Impulsive Control of Financial Risk Dynamic System with Chaotic Characteristics
}

\author{
Li Ziyan $\mathbb{D}^{1},{ }^{1}$ Tao Ke, ${ }^{2}$ Xia Qing $\mathbb{D}^{3},{ }^{3}$ Xie Chengrong, ${ }^{4}$ and Xu Yuhua ${ }^{2}$ \\ ${ }^{1}$ School of Politics and Public Administration, Soochow University, Suzhou 215000, China \\ ${ }^{2}$ School of Finance, Nanjing Audit University, Nanjing 211815, Jiangsu, China \\ ${ }^{3}$ School of Economics, Wuhan Donghu University, Wuhan 430212, China \\ ${ }^{4}$ School of Statistics and Mathematics, Nanjing Audit University, Nanjing 211815, Jiangsu, China \\ Correspondence should be addressed to Xia Qing; 77218434@qq.com
}

Received 10 April 2021; Revised 5 May 2021; Accepted 31 May 2021; Published 10 June 2021

Academic Editor: Xiaodi Li

Copyright (c) 2021 Li Ziyan et al. This is an open access article distributed under the Creative Commons Attribution License, which permits unrestricted use, distribution, and reproduction in any medium, provided the original work is properly cited.

\begin{abstract}
The control of financial risk has always been one of the important topics in financial research. Based on the theory of finance, this paper proposes a kind of financial risk dynamic system. By analyzing some properties of the dynamic system, the system shows obvious coexisting chaotic oscillations. In order to control the financial risk dynamic system effectively, this paper proposes a finite-time impulse controller to control the financial risk dynamic system. Simulation results show that the finite-time impulse controller has faster convergence speed than the impulse controller.
\end{abstract}

\section{Introduction}

Since Lorenz discovered the strange attractor for the first time, various chaotic systems have been discovered one after another. Although there are a large number of chaotic systems, the development of chaotic dynamic models is relatively slow in the economic field [1]. Akhmet et al. investigated the generation of chaos in economic models through exogenous shocks [2]. Liu discussed the two-stage multiplier acceleration econometric model of the capitalist market and the investment model depicting the growth of socialist planned economy. Liu described the instability of the market economy, the cyclical change, and even chaos of the central planned socialist economy [3]. Peters, an American scholar, puts forward the fractal market hypothesis, which makes up for the serious deficiency of the efficient market hypothesis. In particular, it focused on the analysis of the impact of market liquidity and investment duration on investors' behavior, which directly established a model for the changes of investment behavior and price [4]. Liu and Wan discussed the bifurcation and control of the real-estate dynamic model based on the famous logistic wormhole model [5]. Du et al. took the two-dimensional dynamic production game model as an example, taking the form of one participant taking control and two participants participating in control at the same time, and successfully realized the control of chaos in the model [6]. $\mathrm{Yu}$ and $\mathrm{Yu}$ studied stability of Bayesian Nash equilibrium of the dynamic Cournot duopoly model [7]. Ma and Wang analyzed the bifurcation behavior of a new financial chaotic system [8]. Ding et al. discussed the bifurcation behavior of a class of nonlinear financial delay systems [9]. $\mathrm{Ma}$ and Bangura discussed the dynamic behavior of the economic system under the condition of parameter change [10]. We can see that most of the researchers discussed the evolution behavior of the discrete economic and financial chaotic dynamic model. In the economic and financial system, the continuous economic and financial dynamic model may better simulate the real economic and financial world in some extent because of the continuity of economic activities.

At present, many scholars had made extraordinary explorations on the control of economic and financial chaotic systems. For example, Du et al. discussed control of economic dynamical systems by adding upper bound or lower bound to state variables, which is different from the chaos stabilization in engineering or physics systems [11]. Wen 
and Yang analyzed the stability of equilibrium solution of the fractional-order chaotic financial system and explored the complexity evolution law of the financial system [12]. Bella and Mattana proposed a suitable policy algorithm to eliminate or control the chaotic dynamics [13]. Sun et al. applied impulse control to the economic chaotic system, paying more attention to the feasibility of the control method. They achieved good results by saving money and improving efficiency through these methods [14].

Generally speaking, the simulation of economic phenomena based on dynamic principles and the effective control of nonlinear financial complex systems is always attracting in real world. In recent years, a variety of methods has been proposed to realize the effective control of nonlinear complex systems. For example, in [15-20], the authors proposed the impulse control method, which greatly saves the control cost. In [21-25], the authors proposed the finitetime control method, which saves the control time. Therefore, the finite-time impulse control method not only saves the control cost but also reduces the control cost. At the same time, it also saves control time, which will be an effective control method for the economic system $[16,26,27]$. Although some effort has been made in this field, the research on the evolution behavior of financial complex dynamic system based on finite-time impulse control has not received enough attention in recent years.

\section{Chaotic Financial Model and its Basic Analysis}

In [28], the author puts forward a three-stage cycle structure financial system risk model:

$$
\left\{\begin{array}{l}
\dot{x}=y z-a x, \\
\dot{y}=x z-b y, \\
\dot{z}=c z-x y
\end{array}\right.
$$

where $x$ represents the total risk value of the system under the external or internal impact of the first stage, $y$ represents the total risk value of the system under the infection effect of the second stage, $z$ represents the value of the system risk control in the third stage, $a$ represents the degree of risk, $b$ represents the degree of control, and $c$ represents the control strength. When $a=4, b=8$, and $c=2$, system (1) shows chaotic phenomenon.

Since external or internal shocks directly affect the intensity of contagion effect, the financial system risk model (1) can be modified as follows:

$$
\left\{\begin{array}{l}
\dot{x}=y z-a x, \\
\dot{y}=x z-b y+\mathrm{d} x, \\
\dot{z}=-x y+c z,
\end{array}\right.
$$

when $a=4, b=1, c=1$, and $d=1$, the system is in a chaotic state. The corresponding pair of attractors is shown in Figure 1. It can be seen that when the initial values of the system are different, the chaotic attractors have rotational symmetry, which can be proved by the transformation invariance of the system equation. When $x \longrightarrow-x, y \longrightarrow-y$, and $z \longrightarrow z$, the system equation remains unchanged.

Figure 2 shows that the financial system risk model has initial value sensitivity when $a=4, b=1, c=1$, and $d=1$. It can be seen from Figure 2 that the slight change of initial value has a great impact on the solution of the system.

\section{Bifurcation Analysis}

Due to the rotational symmetry of the system and the existence of a pair of coexisting chaotic attractors under the above parameters, the system presents coexistence bifurcation behavior when the system parameters evolve in a certain interval. As shown in Figure 3, when $b=1, c=1$, and $d=1, a$ changes in the interval $[1.57,9.57]$. The system has two bifurcation paths. At the same time, the Lyapunov exponent of the system has the same evolution law. The left plot shows a pair of symmetrical bifurcations under two sets of initial conditions, while the right one shows that coexisting bifurcations obey unified Lyapunov exponents.

When $a=4, c=1$, and $d=1, b$ changes in the interval $[0.1,2.1]$, and the system has two similar bifurcation paths, as shown in Figure 4.

When $a=4, b=1$, and $d=1, c$ changes in the interval $[0.75,3]$, and the system has two similar bifurcation paths, as shown in Figure 5.

It is interesting that the parameter $d$ of system (1) can vary in a large range of positive and negative ranges when the system presents different chaotic motions. Figure 6 shows the bifurcation diagram and Lipschitz exponent spectrum of the system when $d$ changes in the interval $[-25,25]$.

Typically, when $d=0,-1,-10,-12,-18,20$, the solution of the system is shown in Figure 7.

In system (1), the $x$ feedback term of the second dimension can be negative. When $b=1, c=1$, and $d=-1$, the bifurcation diagram and Lipschitz exponent spectrum of the system are shown in Figure 8. It can be seen that the system also presents a pair of coexisting bifurcations.

The above analysis shows that system (2) has the property of coexisting chaos, which brings great risk in real financial world. Therefore, in the following section, an effective method is explored to control the stability of system (2). Among many schematics, we select finite-time impulsive control, which is one of the effective methods to save control cost [13].

\section{Finite-Time Impulsive Control of Chaotic Financial System}

Lemma 1 (see [26]). For $\delta_{i} \geq 0, i=1, \ldots, n, 0<\varsigma \leq 1$, there are

$$
\sum_{i=1}^{n} \delta_{i}^{\varsigma} \geq\left(\sum_{i=1}^{n} \delta_{i}\right)^{\varsigma}
$$

Lemma 2 (see [29]). Suppose a continuous positive definite function satisfies the following differential inequalities: 


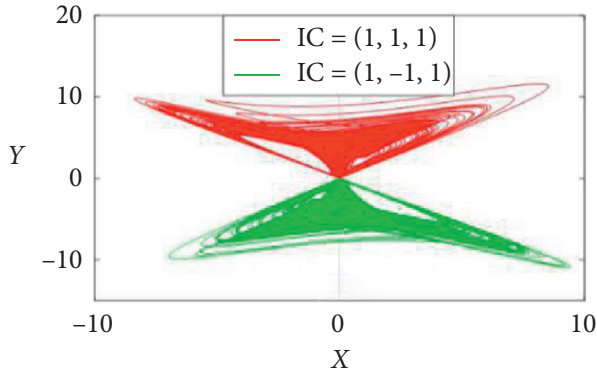

(a)

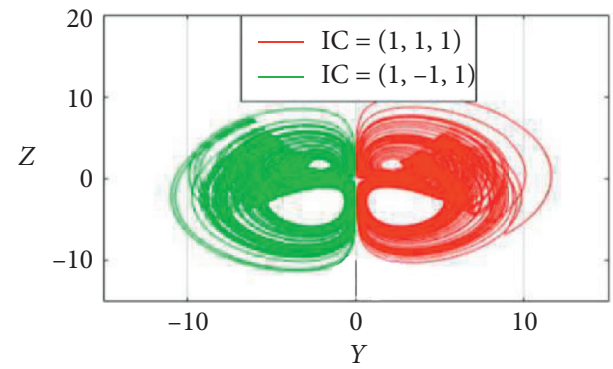

(b)

Figure 1: Projections of chaotic attractors from system (1) with $a=4, b=1, c=1$, and $d=1$ : (a) plane $x-y$; (b) plane $y-z$. IC represents the initial condition.

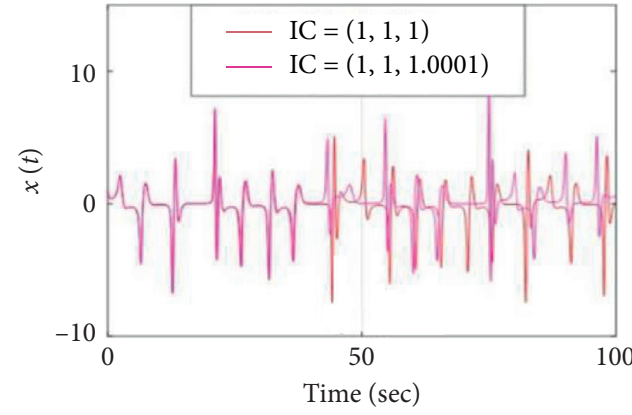

(a)

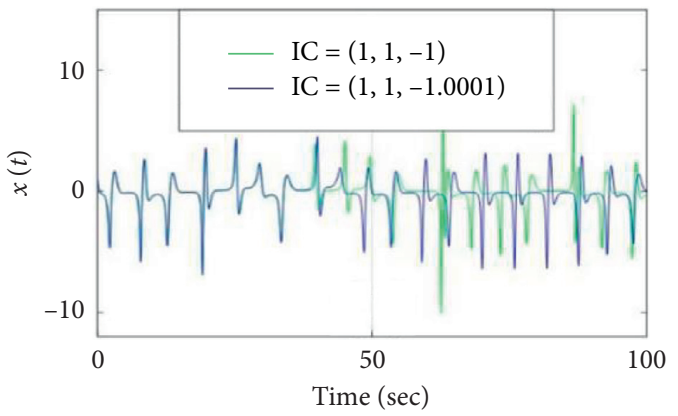

(b)

FIGURE 2: Initial value sensitivity of system (2) with $a=4, b=1, c=1$, and $d=1$ : (a) $x(t)$ in positive attractor and (b) $x(t)$ in negative attractor.

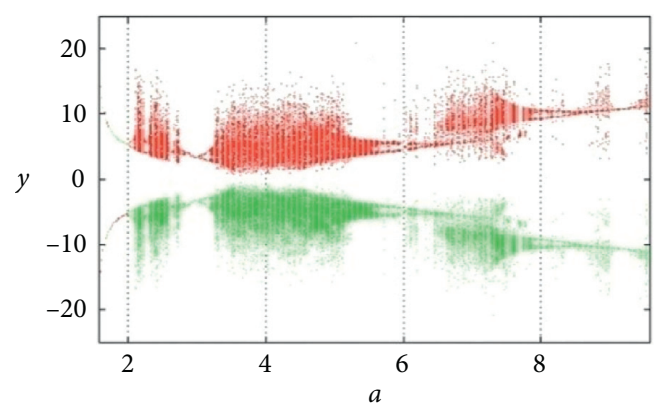

(a)

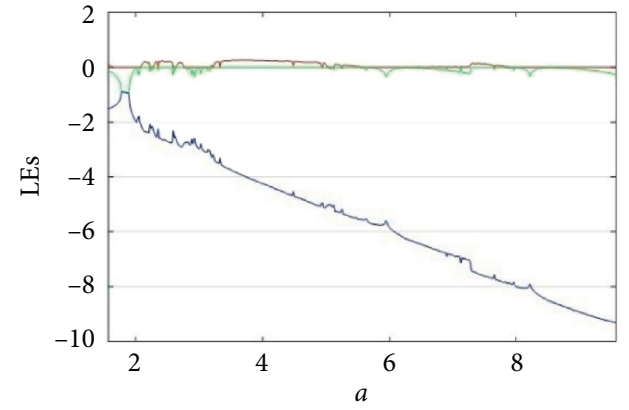

(b)

Figure 3: Dynamical behavior of system (1) with $b=1, c=1$, and $d=1$, and $a$ varies in [1.57, 9.57]: (a) bifurcation diagram $(z=0)$, $\left(x_{0}, y_{0}\right.$, $\left.z_{0}\right)=(1,1,1)$ is red, and $\left(x_{0}, y_{0}, z_{0}\right)=(-1,-1,1)$ is green; (b) Lyapunov exponents (LEs).

$$
\begin{aligned}
\dot{v}(t) & \leq-k v^{\eta}(t), \\
v\left(t_{0}\right) & \geq 0, \quad \forall t \geq t_{0}, k>0,0<\eta<1 .
\end{aligned}
$$

$$
v(t) \leq e^{l\left(t-t_{0}\right)}\left(v^{1-\eta}\left(t_{0}\right)+\frac{k}{l}-\frac{k}{l} e^{l(1-\eta)\left(t-t_{0}\right)}\right)^{1 /(1-\eta)},
$$

Then, for any given $t_{0}, v(t)$ satisfies the following inequality: and $v(t)=0, t \geq T$ with $T$ is given by 


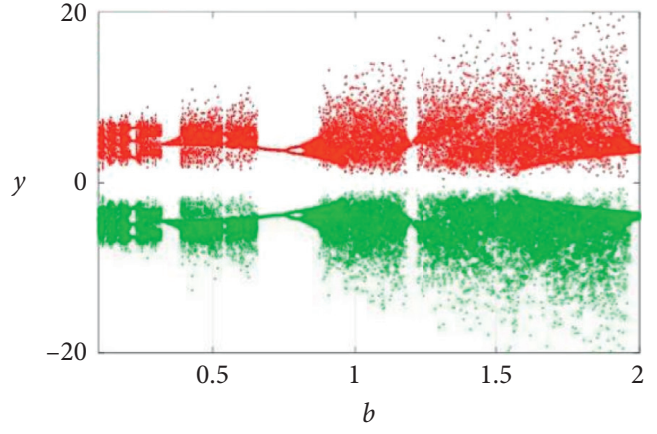

- IC $=(1,1,1)$

$-\mathrm{IC}=(-1,-1,1)$

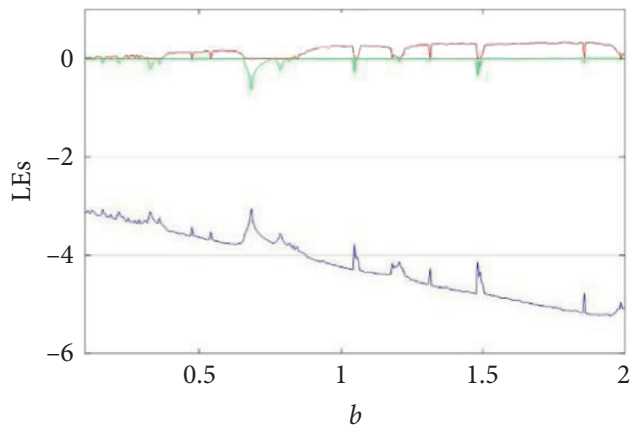

(b)

Figure 4: Dynamical behavior of system (1) with $a=4, c=1$, and $d=1$, and $b$ varies in $[0.1,2.1]$ : (a) bifurcation diagram $(z=0)$, $\left(x_{0}, y_{0}\right.$, $\left.z_{0}\right)=(1,1,1)$ is red, and $\left(x_{0}, y_{0}, z_{0}\right)=(-1,-1,1)$ is green; (b) Lyapunov exponents.

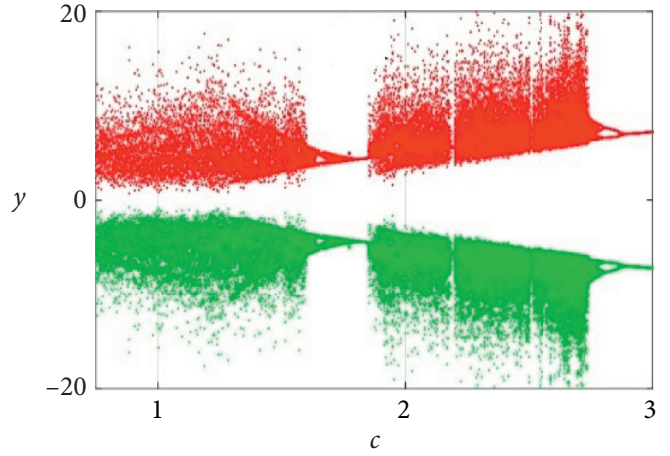

(a)

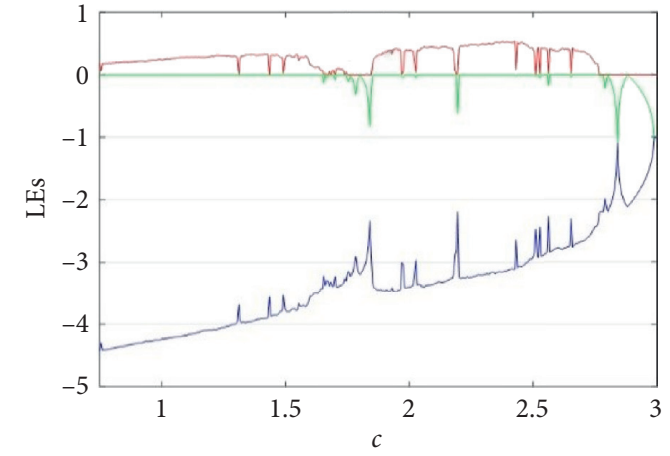

(b)

Figure 5: Dynamical behavior of system (1) with $a=4, b=1$, and $d=1$, and $c$ varies in $[0.75,3]$ : (a) bifurcation diagram $(z=0)$, $\left(x_{0}, y_{0}\right.$, $\left.z_{0}\right)=(1,1,1)$ is red, and $\left(x_{0}, y_{0}, z_{0}\right)=(-1,-1,1)$ is green; (b) Lyapunov exponents.

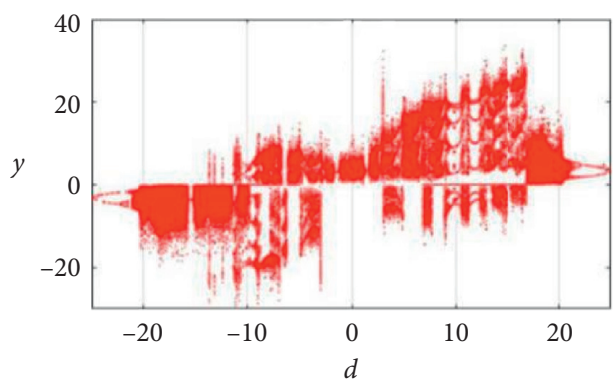

(a)

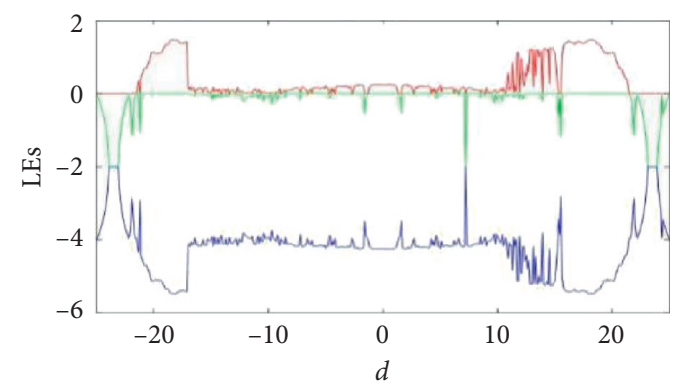

(b)

FIGURe 6: Dynamical behavior of system (1) with $a=4, b=1$, and $c=1,\left(x_{0}, y_{0}, z_{0}\right)=(1,1,1)$, and $d$ varies in [-25, 25]: (a) bifurcation diagram $(x=0)$; (b) Lyapunov exponents. 

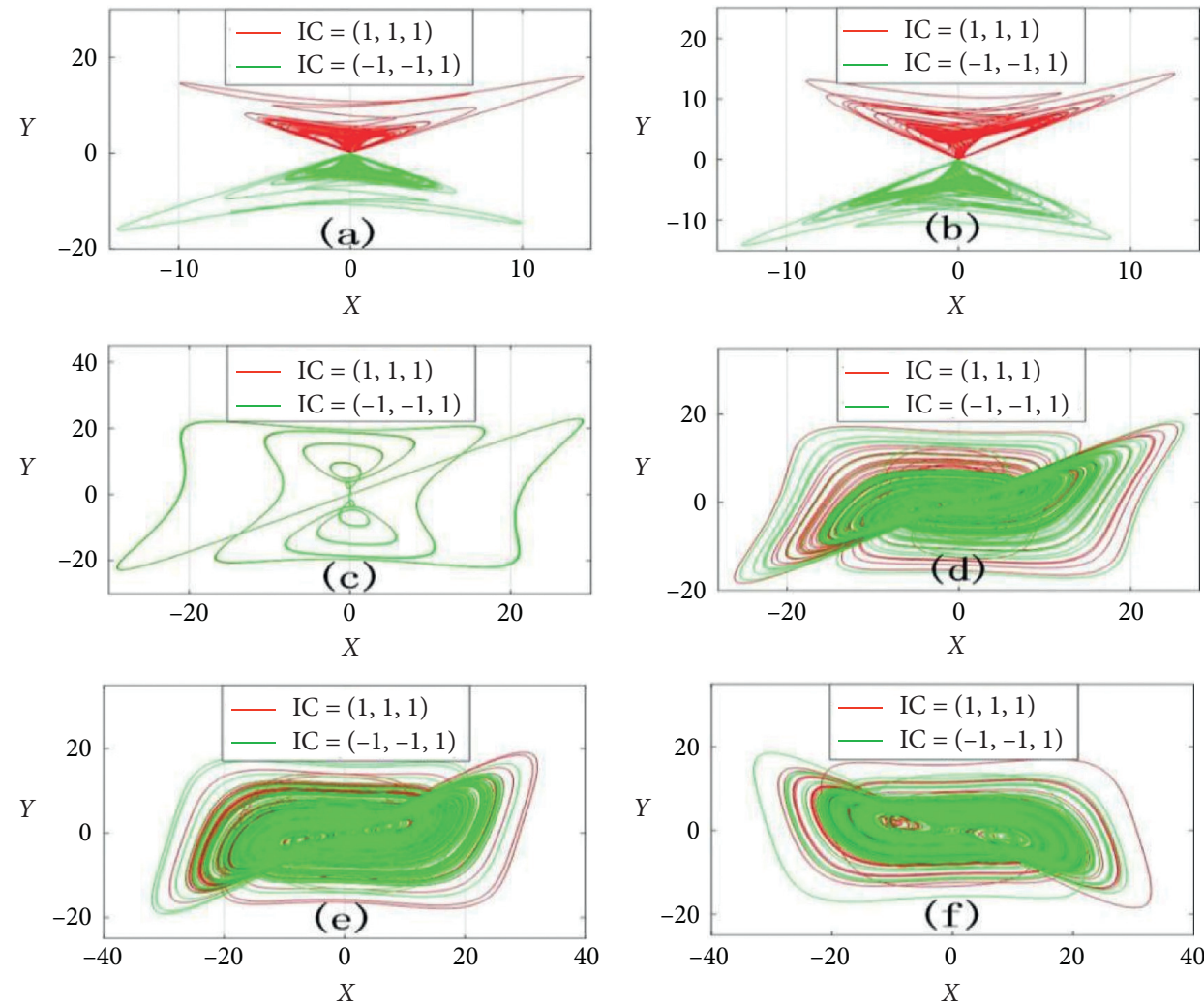

Figure 7: Typical attractors of system (1) with $a=4, b=1$, and $c=1$ : (a) $d=0$, (b) $d=-1$, (c) $d=-10$, (d) $d=-12$, (e) $d=-18$, and (f) $d=20$.

$$
T \leq t_{0}+\frac{v^{1-\eta}\left(t_{0}\right)}{l(1-\eta)} .
$$

Remark 1. For an impulsive system, if it is stable in a finite time, we call it impulsive stable in a finite time.

We consider coupled impulsive control of the chaotic financial system as follows:

$$
\begin{aligned}
& \begin{cases}\dot{x}_{1}=x_{2} x_{3}-a x_{1}+u_{1}, & t \neq t_{k}, \\
\dot{x}_{2}=x_{1} x_{3}-b x_{2}+\mathrm{d} x_{1}+u_{2}, & t \neq t_{k}, \\
\dot{x}_{3}=-x_{1} x_{2}+c x_{3}+u_{3}, & t \neq t_{k}, \\
\Delta x=B(x-y)=-B w, & t=t_{k} .\end{cases} \\
& \begin{cases}\dot{y}_{1}=y_{2} y_{3}-a y_{1}+u_{1}, & t \neq t_{k}, \\
\dot{y}_{2}=y_{1} y_{3}-b y_{2}+\mathrm{d} y_{1}+u_{2}, & t \neq t_{k}, \\
\dot{y}_{3}=-y_{1} y_{2}+c y_{3}+u_{3}, & t \neq t_{k}, \\
\Delta y=B(y-x)=B w, & t=t_{k},\end{cases}
\end{aligned}
$$

where $x=\left(x_{1}, x_{2}, x_{3}\right)^{T} \in R^{3}$ and $y=\left(y_{1}, y_{2}, y_{3}\right)^{T} \in R^{3}$.

The finite-time controller is

$$
\left\{\begin{array}{l}
u_{1}=x_{2} x_{3}-y_{2} y_{3}-k w_{1}-l \operatorname{sgn}\left(w_{1}\right) w_{1}^{\delta}, \\
u_{2}=x_{1} x_{3}-y_{1} y_{3}-k w_{2}-l \operatorname{sgn}\left(w_{2}\right) w_{2}^{\delta}, \\
u_{3}=y_{1} y_{2}-x_{1} x_{2}-k w_{3}-l \operatorname{sgn}\left(w_{3}\right) w_{3}^{\delta},
\end{array}\right.
$$

where $w=\left(w_{1}, w_{2}, w_{3}\right)^{T}=\left(y_{1}-x_{1}, y_{2}-x_{2}, y_{3}-x_{3}\right)^{T}$ and $0<\delta<1$.

So, the error impulsive systems is

$$
\begin{cases}\dot{w}_{1}=y_{2} y_{3}-x_{2} x_{3}-a w_{1}+u_{1}, & t \neq t_{k}, \\ \dot{w}_{2}=y_{1} y_{3}-x_{1} x_{3}-b w_{2}+\mathrm{d} y_{1}+u_{2}, & t \neq t_{k}, \\ \dot{w}_{3}=-y_{1} y_{2}+x_{1} x_{2}+c w_{3}+u_{3}, & t \neq t_{k}, \\ \Delta w=2 B w, & t=t_{k},\end{cases}
$$

where $w=\left(w_{1}, w_{2}, w_{3}\right)^{T}=\left(y_{1}-x_{1}, y_{2}-x_{2}, y_{3}-x_{3}\right)^{T}$.

The objective is to find some conditions on the control gains, $B$, and the impulsive distances $t_{k}-t_{k-1}, k=1,2, \ldots$, such that the impulsive system (9) is asymptotical stable.

Theorem 1. If there exists two constants $\alpha \geq 1, \rho>0$, and $\beta=\lambda_{\max }\left[(I+2 B)^{T}(I+2 B)\right]$, then the error impulsive system (9) can realize finite-time stable under impulsive condition $\ln \alpha \beta+\rho\left(t_{k}-t_{k-1}\right) \leq 0$.

Proof. Let

$$
v(w)=w^{T} w=w_{1}^{2}+w_{2}^{2}+w_{3}^{2} .
$$

The time derivative along trajectory (9) is 


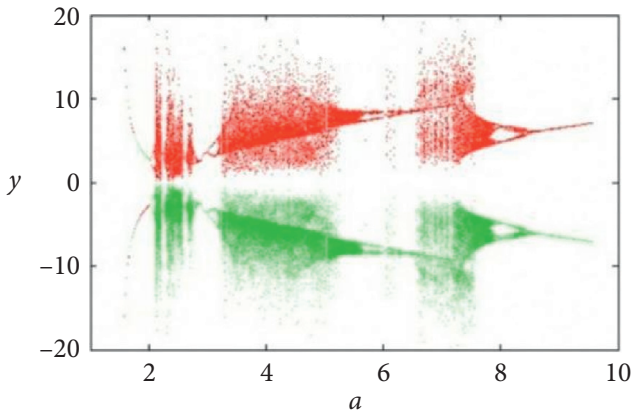

(a)

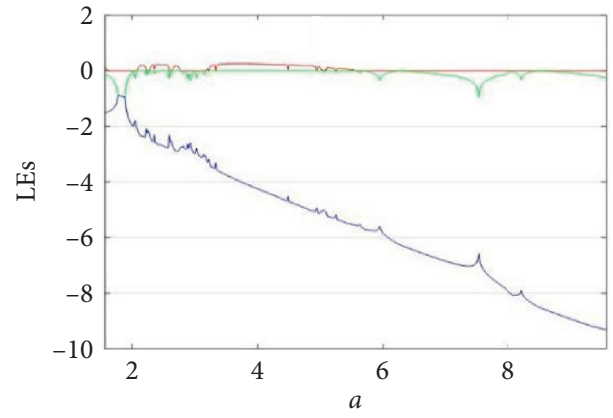

(b)

FiguRe 8: Dynamical behavior of system (1) with $b=1, c=1$, and $d=-1$, and $a$ varies in [1.57, 9.57]: (a) bifurcation diagram $(z=0)$, $\left(x_{0}, y_{0}\right.$, $\left.z_{0}\right)=(1,1,1)$ is red, and $\left(x_{0}, y_{0}, z_{0}\right)=(-1,-1,1)$ is green; (b) Lyapunov exponents.

$$
\begin{aligned}
\dot{v}(w)= & 2 \dot{w}_{1} w_{1}+2 \dot{w}_{2} w_{2}+2 \dot{w}_{3} w_{3} \\
= & 2 w_{1}\left[-a w_{1}-k w_{1}-l \operatorname{sgn}\left(w_{1}\right) w_{1}^{\delta}\right] \\
& +2 w_{2}\left[-b w_{2}-k w_{2}-l \operatorname{sgn}\left(w_{2}\right) w_{2}^{\delta}\right] \\
& +2 w_{3}\left[c w_{3}-k w_{3}-l \operatorname{sgn}\left(w_{3}\right) w_{3}^{\delta}\right] \\
\leq & -2(a+k) w_{1}^{2}-2(b+k) w_{2}^{2}-2(k-c) w_{3}^{2} \leq \rho v(w(t)),
\end{aligned}
$$

where $\rho=\max \{a+k, b+k, k-c\}$.

So,

$$
\begin{aligned}
v(w(t)) & \leq v\left(w\left(t_{k-1}^{+}\right)\right) \exp \left(\rho\left(t-t_{k-1}\right)\right), \\
& t \in\left(t_{k-1}, t_{k}\right], k=1,2, \ldots, \\
v\left(w\left(t_{k}^{+}\right)\right) & =[(I+B) w]^{T}(I+2 B) w \\
& =w^{T}\left[(I+2 B)^{T}(I+2 B)\right] w \leq \beta w^{T} w=\beta v\left(w\left(t_{k}\right)\right) .
\end{aligned}
$$

For $t \in\left(t_{0}, t_{1}\right], v(w(t)) \leq v\left(w\left(t_{0}^{+}\right)\right) \exp \left(\rho\left(t-t_{0}\right)\right)$; then,

$$
v\left(w\left(t_{1}\right)\right) \leq v\left(w\left(t_{0}^{+}\right)\right) \exp \left(\rho\left(t_{1}-t_{0}\right)\right) .
$$

So,

$$
v\left(w\left(t_{1}^{+}\right)\right) \leq \beta v\left(w\left(t_{1}\right)\right) \leq \beta v\left(w\left(t_{0}^{+}\right)\right) \exp \left(\rho\left(t_{1}-t_{0}\right)\right) .
$$

In the same way, for $t \in\left(t_{1}, t_{2}\right]$, we have

$$
\begin{aligned}
v(w(t)) & \leq v\left(w\left(t_{1}^{+}\right)\right) \exp \left(\rho\left(t-t_{1}\right)\right) \\
& \leq \beta v\left(w\left(t_{0}^{+}\right)\right) \exp \left(\rho\left(t-t_{0}\right)\right) .
\end{aligned}
$$

In general, for any $t \in\left(t_{k}, t_{k+1}\right]$, one finds that

$$
v(w(t)) \leq v\left(w\left(t_{0}^{+}\right)\right) \beta^{k} \exp \left(\rho\left(t-t_{0}\right)\right) .
$$

So, $\forall t \in\left(t_{k}, t_{k+1}\right], k=1,2, \ldots$, and we have

$$
\begin{aligned}
v(w(t)) \leq & v\left(w\left(t_{0}^{+}\right)\right) \beta^{k} \exp \left(\rho\left(t-t_{0}\right)\right) \\
\leq & v\left(w\left(t_{0}^{+}\right)\right) \beta^{k} \exp \left(\rho\left(t_{k+1}-t_{0}\right)\right) \\
= & v\left(w\left(t_{0}^{+}\right)\right) \beta \exp \left(\rho\left(t_{2}-t_{1}\right)\right) \beta \exp \left(\rho\left(t_{3}-t_{2}\right)\right) \\
& \cdots \beta \exp \left(\rho\left(t_{k+1}-t_{k}\right)\right) \exp \left(\rho\left(t-t_{0}\right)\right) .
\end{aligned}
$$

As

$$
\beta \exp \left(\mu\left(t_{k+1}-t_{k}\right)\right) \leq \frac{1}{\alpha}, \quad k=1,2, \ldots,
$$

we have

$$
v(w(t)) \leq v\left(w\left(t_{0}^{+}\right)\right) \frac{1}{\alpha^{k}} \exp \left(\rho\left(t-t_{0}\right)\right)
$$

so

$$
v(w(t)) \leq v\left(w\left(t_{0}^{+}\right)\right) \frac{1}{\alpha^{k}} \exp \left(\rho\left(t-t_{0}\right)\right), \quad t \geq t_{0} .
$$

For $\alpha \geq 1$, from [30], it implies that the origin in system (9) is impulsive stable.

On the contrary,

$$
\begin{aligned}
\dot{v}(w)= & 2 \dot{w}_{1} w_{1}+2 \dot{w}_{2} w_{2}+2 \dot{w}_{3} w_{3} \\
= & 2 w_{1}\left[-a w_{1}-k w_{1}-l \operatorname{sgn}\left(w_{1}\right) w_{1}^{\delta}\right] \\
& +2 w_{2}\left[-b w_{2}-k w_{2}-l \operatorname{sgn}\left(w_{2}\right) w_{2}^{\delta}\right] \\
& +2 w_{3}\left[c w_{3}-k w_{3}-l \operatorname{sgn}\left(w_{3}\right) w_{3}^{\delta}\right] \\
= & -2(a+k) w_{1}^{2}-2(b+k) w_{2}^{2}-2(k-c) w_{3}^{2} \\
& -2 l w_{1} \operatorname{sgn}\left(w_{1}\right) w_{1}^{\delta}-2 l w_{2} \operatorname{sgn}\left(w_{2}\right) w_{2}^{\delta} \\
& -2 l w_{3} \operatorname{sgn}\left(w_{3}\right) w_{3}^{\delta} \\
\leq & -2 l w_{1} \operatorname{sgn}\left(w_{1}\right) w_{1}^{\delta}-2 l w_{2} \operatorname{sgn}\left(w_{2}\right) w_{2}^{\delta} \\
& -2 l w_{3} \operatorname{sgn}\left(w_{3}\right) w_{3}^{\delta} \\
= & -2 l\left(\left|w_{1}\right|^{\delta+1}+\left|w_{2}\right|^{\delta+1}+\left|w_{2}\right|^{\delta+1}\right) .
\end{aligned}
$$

From Lemma 1,

$$
\begin{aligned}
& \left(\left|w_{1}\right|^{\delta+1}+\left|w_{2}\right|^{\delta+1}+\left|w_{2}\right|^{\delta+1}\right) \\
& \quad=\left(\left(w_{1}^{2}\right)^{(\delta+1 / 2)}+\left(w_{2}^{2}\right)^{(\delta+1 / 2)}+\left(w_{3}^{2}\right)^{(\delta+1 / 2)}\right) \\
& \geq\left(w_{1}^{2}+w_{2}^{2}+w_{3}^{2}\right)^{(\delta+1 / 2)},
\end{aligned}
$$




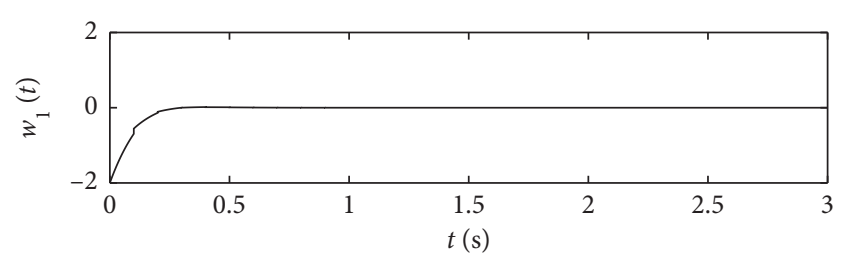

(a)

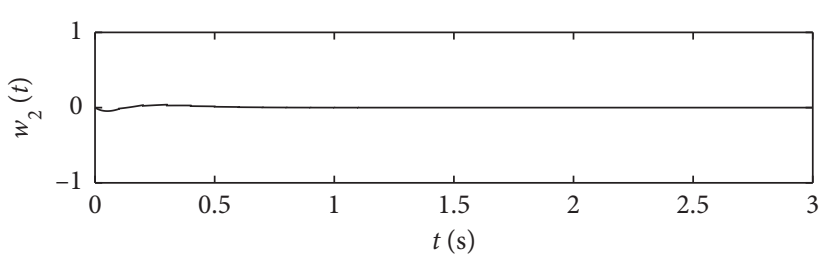

(b)

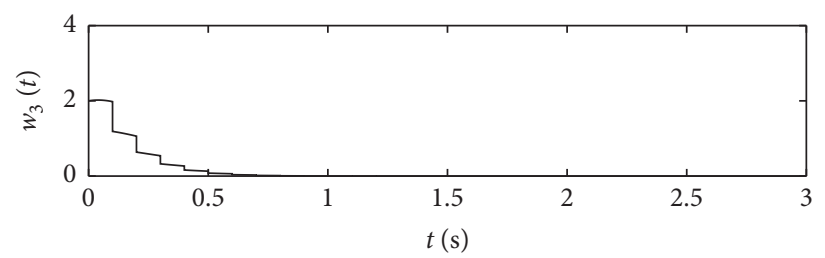

(c)

FIGURE 9: Finite-time impulsive synchronization errors.

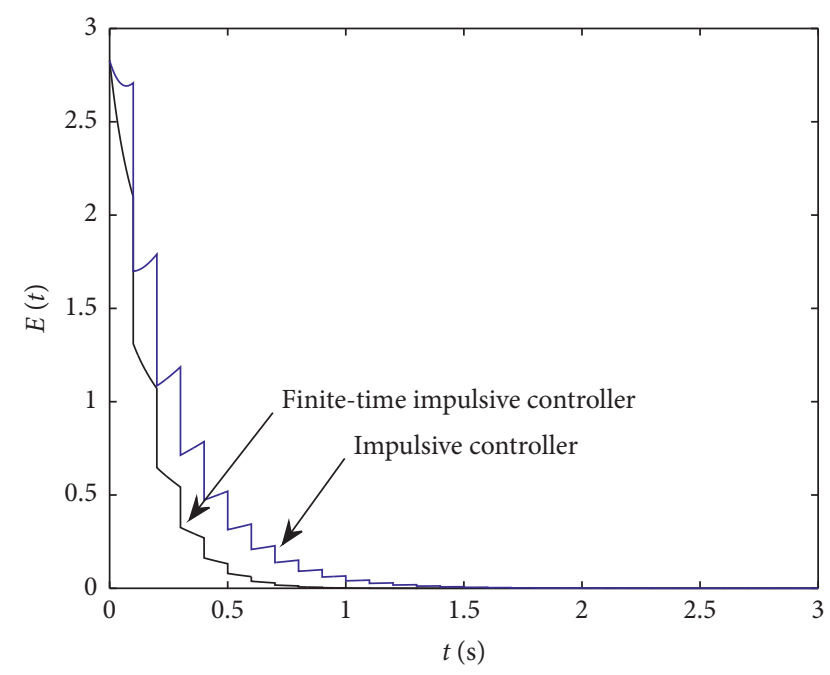

FIGURE 10: Comparison between finite-time impulsive controller and impulsive controller.

$$
\dot{v}(w) \leq-2 l\left(w_{1}^{2}+w_{2}^{2}+w_{3}^{2}\right)^{(\delta+1 / 2)}=-2 l v^{(\delta+1 / 2)} .
$$

From Lemma 2, system (9) is finite-time stable.

To sum up, system (9) can realize finite-time stable under impulsive condition.

Remark 2. In $[15,16,18]$, the authors put forward the principle of impulsive control in nonlinear systems, and their conclusions provide the basic theoretical foundation for this paper. However, the exact finite-time impulsive controller of financial systems needs to be designed.

In the following simulation, we set $a=4, b=1, c=1$, $d=1, B=\operatorname{diag}(-0.2,-0.3,-0.4), \rho=6, k=2$, and $l=1$; then, $\beta=0.36$. Let $\alpha=1.2$, from $\ln \alpha \beta+\rho\left(t_{k}-t_{k-1}\right) \leq 0$; then, $t_{k}-t_{k-1} \leq 0.1399$ and we let $t_{k}-t_{k-1}=0.1$. The initial conditions of the master and slave systems are $(1,2,3)$ and $(3,2,1)$. In Figure 9, three state errors versus time are shown and the state errors tend to zero asymptotically as time evolves under finite-time impulsive controller. Let $E(t)=\sqrt{w_{1}^{2}+w_{2}^{2}+w_{3}^{2}}$; Figure 10 shows the finite-time impulsive controller has faster convergence speed than impulsive controller.

\section{Conclusion and Discussion}

The paper has presented a new chaotic financial system and discussed some basic dynamic behavior of the new system. Although many approaches have been reported for chaos control, many of them are associated with amplitude control $[31,32]$ and offset boosting [33-35] without removing the inherent chaos. In this work, based on finite-time control theory, the finite-time impulsive synchronization criterion of the new chaotic financial system has been studied and proposed. The results of numerical simulation prove that they are feasible for the new financial system, and finite-time impulse control of the economic system provides a helpful reference scheme for real economic world. As the coexisting chaos-embedded bifurcations of economic system brings great risk to the economy, if the bifurcation behavior of economic system can be controlled by the finite-time impulse method, the instability of the system will be reduced, which will be our next research topic.

\section{Data Availability}

No data were used to support this study.

\section{Conflicts of Interest}

The authors declare that there are no conflicts of interest regarding the publication of this paper.

\section{Acknowledgments}

The authors are grateful to J. C. Sprott for helping with parameter selection that led to this work. This work was supported by the National Natural Science Foundation of China (61871230), the Major Natural Science Foundation of Jiangsu Higher Education Institutions (20KJA120002), the 
Natural Science Foundation of Jiangsu Higher Education Institutions (19KJB120007), the Natural Science Foundation of Jiangsu Province (BK20181418), and the Six Talent Peaks Project in Jiangsu Province (DZXX-019).

\section{References}

[1] Y. Xu, Z. Ke, W. Zhou, and C. Xie, "Dynamic evolution analysis of stock price fluctuation and its control," Complexity, vol. 2018, Article ID 5728090, 9 pages, 2018.

[2] M. Akhmet, Z. Akhmetova, and M. O. Fen, "Chaos in economic models with exogenous shocks," Journal of Economic Behavior \& Organization, vol. 106, pp. 95-108, 2014.

[3] H. Liu, Chaos Theory and Method of Economic System Prediction, Science Press, Beijing, China, 2003.

[4] E. E. Peters, Fractal Market Analysis: Applying Chaos Theory to Investment and Economics, Wiley Finance, Hoboken, NJ, USA, 1994.

[5] J. Liu and W. Han, "OGY chaos control strategy for real estate investment bubble," Systems Engineering, vol. 3, pp. 41-43, 2002.

[6] J. Du, Z. Sheng, and H. Yao, "Threshold control method for a class of chaotic economic model," Systems Engineering-Theory \& Practice, vol. 10, pp. 27-32, 2004.

[7] W. Yu and Y. Yu, "The stability of Bayesian Nash equilibrium of dynamic cournot duopoly model with asymmetric information," Communications in Nonlinear Science and Numerical Simulation, vol. 63, pp. 101-116, 2018.

[8] C. Ma and X. Wang, "Hopf bifurcation and topological horseshoe of a novel finance chaotic system," Communications in Nonlinear Science and Numerical Simulation, vol. 17, no. 2, pp. 721-730, 2012.

[9] Y. Ding, W. Jiang, and H. Wang, "Hopf-pitch fork bifurcation and periodic phenomenain nonlinear financial system with delay," Chaos, Solitons \& Fractals, vol. 45, no. 8, pp. 1048-1057, 2012.

[10] J. Ma and H. I. Bangura, "Complexity analysis research of financial and economic system under the condition of three parameters' change circumstances," Nonlinear Dynamics, vol. 70, no. 4, pp. 2313-2326, 2012.

[11] J. Du, T. Huang, Z. Sheng, and H. Zhang, "A new method to control chaos in an economic system," Applied Mathematics and Computation, vol. 217, no. 6, pp. 2370-2380, 2010.

[12] C. Wen and J. Yang, "Complexity evolution of chaotic financial systems based on fractional calculus," Chaos, Solitons \& Fractals, vol. 128, pp. 242-251, 2019.

[13] G. Bella and P. Mattana, "Chaos control in presence of financial bubbles," Economics Letter, vol. 193, Article ID 109314, 2020.

[14] J. Sun, F. Qiao, and Q. Wu, "Impulsive control of a financial model," Physics Letters A, vol. 335, no. 4, pp. 282-288, 2005.

[15] X. Li, X. Yang, and T. Huang, "Persistence of delayed cooperative models: impulsive control method," Applied Mathematics and Computation, vol. 342, pp. 130-146, 2019.

[16] X. Li, J. Shen, and R. Rakkiyappan, "Persistent impulsive effects on stability of functional differential equations with finite or infinite delay," Applied Mathematics and Computation, vol. 329, pp. 14-22, 2018.

[17] X. Li, J. Shen, H. Akca, and R. Rakkiyappan, "LMI-based stability for singularly perturbed nonlinear impulsive differential systems with delays of small parameter," Applied Mathematics and Computation, vol. 250, pp. 798-804, 2015.
[18] R. Rao and S. Zhong, "Impulsive control on delayed feedback chaotic financial system with Markovian jumping," Advances in Difference Equations, vol. 2020, no. 1, 2020.

[19] P. Li, X. Li, and J. Lu, "Input-to-state stability of impulsive delay systems with multiple impulses," IEEE Transactions on Automatic Control, vol. 66, no. 1, pp. 362-368, 2020.

[20] Y. Wang, J. Lu, and Y. Lou, "Halanay-type inequality with delayed impulses and its applications," Science China Information Sciences, vol. 62, no. 9, Article ID 192206, 2019.

[21] Y. Xu, X. Wu, N. Li, L. Liu, C. Xie, and C. Li, "Fixed-time synchronization of complex networks with a simpler nonchattering controller," IEEE Transactions on Circuits and Systems II: Express Briefs, vol. 67, no. 4, pp. 700-704, 2020.

[22] Y. Xu, X. Wu, B. Mao, and C. Xie, "A unified finite-/fixed-time synchronization approach to multi-layer networks," IEEE Transactions on Circuits and Systems II: Express Briefs, vol. 68, no. 1, pp. 311-315, 2021.

[23] Y. Xu, X. Wu, B. Mao, J. Lü, and C. Xie, "Fixed-time synchronization in pth moment for time-varying delay stochastic multilayer networks," IEEE Transactions on Systems, Man, and Cybernetics: Systems, vol. 99, pp. 1-10, 2020.

[24] Y. Xu, X. Wu, B. Mao, J. Lü, and C. Xie, "Finite-time intralayer and inter-layer quasi-synchronization of two-layer multi-weighted networks," IEEE Transactions on Circuits and Systems I: Regular Papers, vol. 68, no. 4, pp. 1589-1598, 2021.

[25] J. Lu, Y. Wang, X. Shi, and J. Cao, "Finite-time bipartite consensus for multi-agent systems under detail-balanced antagonistic interactions," IEEE Transactions on Systems, Man, and Cybernetics: Systems, vol. 51, no. 6, pp. 3867-3875, 2021.

[26] X. Li, D. W. C. Ho, and J. Cao, "Finite-time stability and settling-time estimation of nonlinear impulsive systems," Automatica, vol. 99, pp. 361-368, 2019.

[27] Q. Xi, Z. Liang, and X. Li, "Uniform finite-time stability of nonlinear impulsive time-varying systems," Applied Mathematical Modelling, vol. 91, pp. 913-922, 2021.

[28] Y. Xu, C. Xie, and Y. Wang, "Evolution mechanism of financial system risk," Statistics and Decision Making, vol. 1, pp. 172-175, 2016.

[29] Y. Xu, W. Zhou, J. a. Fang, C. Xie, and D. Tong, "Finite-time synchronization of the complex dynamical network with nonderivative and derivative coupling," Neurocomputing, vol. 173, pp. 1356-1361, 2016.

[30] B. Jiang, J. Lu, and Y. Liu, "Exponential stability of delayed systems with average-delay impulses," SIAM Journal on Control and Optimization, vol. 58, no. 6, pp. 3763-3784, 2020.

[31] C. Li, J. Clinton Sprott, A. Akgul, H. H. C. Iu, and Y. Zhao, "A new chaotic oscillator with free control," Chaos, vol. 27, Article ID 083101, 2017.

[32] C. Li, W. Joo-Chen Thio, H. Ho-Ching Iu, and T. Lu, “A memristive chaotic oscillator with increasing amplitude and frequency," IEEE Access, vol. 6, pp. 12945-12950, 2018.

[33] C. Li, T. Lei, X. Wang, and G. Chen, "Dynamics editing based on offset boosting," Chaos, vol. 30, Article ID 063124, 2020.

[34] T. Lu, C. Li, X. Wang, C. Tao, and Z. Liu, "A memristive chaotic system with offest-boostable conditional symmetry," European Physical Journal Special Topics, vol. 229, no. 6-7, pp. 1059-1069, 2020.

[35] C. Li, G. Chen, J. Kurths, T. Lei, and Z. Liu, "Dynamic transport: from bifurcation to multistability," Communications in Nonlinear Science and Numerical Simulation, vol. 95, Article ID 105600, 2021. 